\title{
Improved Socio-Economic Status of a Community Population Following Schistosomiasis and Intestinal Worm Control Interventions on Kome Island, North-Western Tanzania
}

\author{
Joseph R. Mwanga', Godfrey M. Kaatano', Julius E. Siza', Su Young Chang², Yunsuk Ko², Cyril M. Kullaya², \\ Jackson Nsabo ${ }^{2}$, Keeseon S. Eom ${ }^{3, *}$, Tai-Soon Yong ${ }^{4}$, Jong-Yil Chai ${ }^{5}$, Duk-Young Min ${ }^{6}$, Han-Jong Rim, \\ John M. Changalucha'
}

${ }^{1}$ National Institute for Medical Research, P.O. Box 1462, Mwanza, Tanzania; '2Good Neighbors International, Tanzania Western Chapter, P.O. Box 367, Mwanza, Tanzania; ${ }^{3}$ Department of Parasitology, Medical Research Institute and Parasite Resource Bank, Chungbuk National University School of Medicine, Cheongju 28644, Korea; ${ }^{4}$ Department of Environmental Medical Biology, Institute of Tropical Medicine and Arthropods of Medical Importance Resource Bank, Yonsei University College of Medicine, Seoul 03722, Korea; ${ }^{5}$ Department of Parasitology and Tropical Medicine, Seoul National University College of Medicine, Seoul 03080, Korea; ${ }^{6}$ Department of Immunology and Microbiology, Eulji University School of Medicine, Daejeon 35233, Korea; 'Department of Parasitology, College of Medicine, Korea University, Seoul 02841, Korea

\begin{abstract}
Research on micro-level assessment of the changes of socio-economic status following health interventions is very scarce. The use of household asset data to determine wealth indices is a common procedure for estimating socioeconomic position in resource poor settings. In such settings information about income is usually lacking, and the collection of individual consumption or expenditure data would require in-depth interviews, posing a considerable risk of bias. In this study, we determined the socio-economic status of 213 households in a community population in an island in the north-western Tanzania before and 3 year after implementation of a participatory hygiene and sanitation transformation (PHAST) intervention to control schistosomiasis and intestinal worm infections. We constructed a household 'wealth index' based housing construction features (e.g., type of roof, walls, and floor) and durable assets ownership (e.g., bicycle, radio, etc.). We employed principal components analysis and classified households into wealth quintiles. The study revealed that asset variables with positive factor scores were associated with higher socio-economic status, whereas asset variables with negative factor scores were associated with lower socio-economic status. Overall, households which were rated as the poorest and very poor were on the decrease, whereas those rated as poor, less poor, and the least poor were on the increase after PHAST intervention. This decrease/increase was significant. The median shifted from -0.4376677 to 0.5001073 , and the mean from -0.2605787 (SD; 2.005688) to 0.2605787 (SD; 1.831199). The difference in socio-economic status of the people between the 2 phases was highly statistically significant $(P<0.001)$. We argue that finding of this study should be treated with caution as there were other interventions to control schistosomiasis and intestinal worm infections which were running concurrently on Kome Island apart from PHAST intervention.
\end{abstract}

Key words: Schistosomiasis, intestinal worm, socio-economic status, control, principal components analysis, participatory hygiene and sanitation transformation (PHAST), Tanzania

\section{INTRODUCTION}

Accurate tools for assessing household wealth are essential for many health studies in developing countries. Theoretically, measures of household wealth can be reflected by income,

- Received 23 July 2015, revised 30 September 2015, accepted 30 September 2015. *Corresponding author (keeseon.eom@gmail.com)

(c) 2015, Korean Society for Parasitology and Tropical Medicine

This is an Open Access article distributed under the terms of the Creative Commons Attribution Non-Commercial License (http://creativecommons.org/licenses/by-nc/3.0) which permits unrestricted non-commercial use, distribution, and reproduction in any

medium, provided the original work is properly cited. consumption, or expenditure information. Consumption is generally accepted as the most accurate and direct measure of household socio-economic status (SES) [1-3]. However, because of high costs involved in implementing consumption surveys in poor-resource settings, alternatively, asset-based indices are the commonest used method [4], which recently has increasingly been used because of its simplicity and lower cost when compared with consumption surveys.

Data reduction techniques like widely used principal components analysis (PCA) [5,6] and principal axis factoring (PAF) [7], or less known multiple correspondence analysis (MCA) 
[8], are increasingly gaining popularity in estimating SES.

This sub-study was a part of a programme aimed at a combined control of neglected tropical diseases (NTDs) on Kome Island and the Lake Victoria basin in Tanzania, funded by Korean International Cooperation Agency (KOICA), through Good Neighbors International (GNI). The main objective of this sub-study was to evaluate the impact of participatory hygiene and sanitation transformation (PHAST) approach on people's SES following schistosomiasis and intestinal worm control interventions. Specifically the study intended to examine changes in people's SES and measure the beneficial effects following locally adapted schistosomiasis and intestinal worm control interventions.

\section{MATERIALS AND METHODS}

\section{Study area and population}

The baseline study was carried out towards the end of 2009 and follow-up study around the same time in 2012 on Kome Island, on the Lake Victoria basin in Tanzania situated in the south-eastern part of Lake Victoria. The island lies between $32^{\circ} 24^{\prime}$ and $32^{\circ} 34^{\prime}$ east, and $2^{\circ} 14^{\prime}$ and $2^{\circ} 25^{\prime}$ south covering a total area of about $290 \mathrm{~km}^{2}$. This Island is a division of Sengerema District of Mwanza region, and it is divided into 2 administrative wards, namely Nyakasasa and Lugata each with 4 villages. Nyakasasa, Isenyi, Buhama, and Nyamiswi villages belong to Nyakasasa ward; and Lugata, Nyakabanga, Bugolo, and Kabaganga villages belong to Lugata ward. The most recent Population and Housing Census of 2012 put the Island's population of 47,534 people of which 24,480 are males and 23,054 are females. The average household size is 5.8 [9].

The island is predominantly inhabited by Wazinza ethnic group (a Bantu-speaking people). Most of the inhabitants earn their living from peasantry and livestock-keeping. They cultivate mainly cassava, sweet potatoes, maize, and paddy, and keep animals mainly cattle, goats, sheep, and poultry. A few of them undertake fishing as an occupation. Crop production and livestock keeping take place within the framework of individual households.

Lake Victoria is the main source of water for domestic, occupational, and recreational activities. Other sources of water are natural wells and streams. All water contact activities of the people on Kome Island have an obvious bearing on acquisition and transmission of intestinal schistosomiasis and other parasitic diseases. The island is served by 1 health center and 2 dispensaries with chronic shortages of personnel, equipments, and drugs. Nevertheless, since 2008 there has been concerted efforts to control schistosomiasis and intestinal worm infections by the GNI through its Tanzania Western Chapter, in collaboration with the National Institute for Medical Research (NIMR), Mwanza Center, through mass treatment of schistosomiasis and intestinal worms with praziquantel and albendazole, respectively, provision of pumped wells in each of subvillage, and community adult health education on schistosomiasis and intestinal worm infections through PHAST strategy. These combined control activities were undertaken for 5 years (2008-2013) under the auspices of a programme called the 'Control of NTDs on Kome Island and the Lake Victoria basin in Tanzania'.

\section{Study design}

This was a quasi-experimental study based on a pre-/postintervention comparison ('before/after' study) and process evaluation in which PHAST intervention was implemented for 3 years among a community of Kome Island. The study population was observed before, during, and after the intervention to test, explore, and explain the dynamics of socio-economic status in the face of an intervention.

\section{PHAST intervention}

The intervention which was introduced to control schistosomiasis and intestinal worm infections among the people on Kome Island is known as PHAST. This initiative was introduced in Tanzania in 1999. It originated from efforts to control cholera and other diarrheal diseases [10]. PHAST is widely regarded as an innovative approach to promote hygiene, sanitation, and community management of water and sanitation facilities. It is an approach to health promotion that was originally intended to focus on community participation and capacity development rather than health messages alone but also inducing improved hygiene behavior change. The underlying principle of PHAST is that no lasting changes in people's behavior will occur without communities internalizing the link between disease and poor hygiene and sanitation. The approach attempts to go beyond the teaching of hygiene and sanitation concepts to developing capacity of participants to identify opportunities and overcome obstacles to change generally. The approach as it was applied on Kome Island was intended to build communal capacity to plan, monitor, and evaluate sustainable hygiene and sanitation programme which 
would be fully owned and controlled by the community. The methodological experience of the PHAST approach in the control of schistosomiasis in Tanzania has been described elsewhere $[11,12]$.

\section{Sampling procedures}

The households involved in this study were randomly selected for asset-based questionnaire surveys before and after introduction of the PHAST intervention. The sample size for the baseline study was 242 households. During the follow-up (post-intervention survey), the sample size dropped to 213 households. Hence, 29 households were lost to follow-up, mainly because heads of households could not be found anymore during the post-intervention survey. Therefore, our cohort consisted of a total of 213 households for which data from the baseline survey and the 3-years post-intervention follow-up were available. However, characteristics of those households that were lost during the follow-up survey were compared with the other households with no significant differences found (e.g., number of household members and wealth quintiles).

\section{Asset-based questionnaires}

Researcher-administered household asset-based questionnaire surveys were conducted (one in November 2009 for baseline and another in December 2012 as follow-up) to assess changes in the people's socio-economic status on Kome Island before and 3-year after implementation of the PHAST intervention to control schistosomiasis and intestinal worms. During the surveys, information about respondents' demographic, social, and economic characteristics, household durable assets, and housing characteristics were collected through a standardized questionnaire, similar in both surveys and with the same respondents (heads of households).

\section{Ethical considerations}

The scientific and ethical clearance for this study was obtained from the national health research ethics body known as the Medical Research Coordinating Committee. An information sheet read/provided to participants categorically stated study objectives, procedures, and potential risks and benefits. Signatures or finger prints (for illiterates) on the consent forms was solicited from participants implying to have consented to participate in the study.

\section{Data management}

Data obtained from the asset-based questionnaire surveys were edited in the field for omission and/or inconsistencies and for quality control were entered by 2 independent data entry clerks using CSPro version 3.3 (US Census Bureau, 2007) computer software for cleaning and validation.

We then constructed an asset index following the method described by Vyas and Kumaranayake [13] as follows: First, we selected 14 asset variables for further analysis taking into consideration of the distribution of assets in question [13]. Asset variables with very low or very high frequencies were dropped. Only those asset variables which depicted wealth (with due respect to the local socio-cultural situation) were included in the analysis. Then, we re-coded qualitative categorical variables into binary variables (with binary variable 0 and 1 representing asset ownership; i.e., $0=$ no, $1=$ yes). The same set of asset variables for the 2 surveys $(n=14)$ were used.

Second, data analysis for the asset index used PCA to discriminate between households in asset ownership, method advocated by Filmer and Pritchett [4]. Each of the selected assets in each survey separately was assigned a weighted sum as proxy for SES by using STATA version 10.1 (Stata Corp., College Station, Texas, USA). Each household in a retained cohort $(\mathrm{n}=213)$ was assigned a standardized score for each asset, where the score differed depending on whether or not the household has that asset.

Third, the resulting asset scores were then standardized in relation to a standard normal distribution with a mean of zero and SD of one. This method assigned a weighted sum as a proxy for socio-economic wealth to each household included in the sample. These category-weights were then applied to the normalized responses of the households, and we ranked them according to their achieved weighted sum in the 2 surveys.

Fourth, subsequently scores were used to create break points that defined population wealth quintiles which were divided into 5 groups as follows: (i) Most Poor; (ii) More Poor; (iii) Poor; (iv) Less poor; and (v) Least poor. Wealth quintiles were expressed in terms of quintiles of individuals in the sample. This approach to defining wealth quintile has the advantage of producing information directly relevant to the primary question of interest. This choice also facilitated comparison across indicators for the same quintile, since the quintile denominator remain unchanged across indicators.

Fifth, summary statistics were then calculated. All statistical tests were carried out at $5 \%$ significance level. The significance 
of the differences in SES of the study households before and after the PHAST intervention was determined by using the non-parametric Wilcoxon signed-rank test for paired data and the Student's $t$-test for paired samples, and significance of the differences in SES was established.

\section{RESULTS}

\section{Socio-demographic characteristics of respondents}

A total of 213 households were retained during the followup survey. Table 1 shows socio-demographic characteristics of the study respondents. The vast majority of our study cohort $(\mathrm{n}=193 ; 90.6 \%)$ were males, whereas the remaining 20 respondents were female-heads of households. Two hundred respondents (93.9\%) were in the age range of 25-74 years. Three-quarters of the respondents $(\mathrm{n}=159 ; 74.6 \%)$ attained primary school education. However, a quarter of the respondents $(\mathrm{n}=53 ; 24.9 \%)$ had no formal education. With regard to religion, the vast majority of the respondents $(\mathrm{n}=181$; $85.0 \%$ ) were Christians. The livelihood of the vast majority of the respondents $(n=206 ; 96.7 \%)$ depended on peasantry.

\section{Improvements in socio-economic status}

Fourteen asset variables which were included in the analysis

Table 1. Socio-demographic characteristics of respondents

\begin{tabular}{lcc}
\hline Sex & Respondents $(n=213)$ & Percentage (\%) \\
\hline Males & 193 & 90.6 \\
Females & 20 & 9.4 \\
Age groups & & \\
15-24 & 5 & 2.4 \\
$25-34$ & 37 & 17.4 \\
$35-44$ & 56 & 26.3 \\
$45-54$ & 45 & 21.1 \\
$55-64$ & 35 & 16.4 \\
$65-74$ & 27 & 12.7 \\
$75+$ & 8 & 3.8 \\
Religion & & \\
Christian & 181 & 85.0 \\
Muslim & 9 & 4.2 \\
Traditional & 23 & 10.8 \\
Level of education & & \\
No formal education & 1 & 0.94 \\
Primary education (Std. VII) & 159 & 0.94 \\
College & 1 & 0.47 \\
Main occupation & 206 & 0.47 \\
Peasantry & 1 & \\
Livestock-keeping & & \\
Petty business & & \\
Fishing & & \\
Employee & & \\
Other & & \\
\hline
\end{tabular}

and their corresponding weights obtained from the PCA are provided in Table 2 . The study revealed that variables with positive factor scores, i.e., houses with floor made of cement (cement floor), walls made of cement bricks (cement block walls), or walls made of burnt bricks, and a roof made of corrugated iron sheets (corrugated iron roof) carried higher weights, and hence were associated with higher SES. Conversely, variables with negative factor scores (i.e. houses with mud floors, walls made of mud (mud wall), and a roof made of grass (thatched roof)) carried lower weights, and hence were associated with lower SES. This observation was true for both baseline and the post-intervention surveys.

The distribution of households in wealth quintiles during the baseline and follow-up surveys is shown in Table 3. The study revealed that, overall, households which were rated as the poorest and very poor were on the decrease, whilst those rated as poor, less poor, and least poor were on the increase between baseline and follow-up phases. This increase/decrease was statistically significant.

Table 2. Items included in asset indices, and their weights

\begin{tabular}{lcc}
\hline Asset variable & $\begin{array}{c}\text { Study population } \\
\text { pooled }(\mathrm{n}=540)\end{array}$ & $\begin{array}{c}\text { Study cohort } \\
(\mathrm{n}=426)\end{array}$ \\
\hline Corrugated iron roof & 0.3844 & 0.3849 \\
Thatched roof & -0.3887 & -0.3892 \\
Cement block wall & 0.2382 & 0.2349 \\
Burnt bricks wall & 0.2998 & 0.3107 \\
Mud wall & -0.3884 & -0.3946 \\
Cement floor & 0.3798 & 0.3918 \\
Mud floor & -0.3671 & -0.3729 \\
Bicycle & 0.1932 & 0.1797 \\
Radio & 0.1326 & 0.1398 \\
Mattress (sponge) & 0.1174 & 0.1015 \\
Cow & 0.1808 & 0.1521 \\
Sheep/goat & 0.1347 & 0.1095 \\
Chicken/duck & 0.0715 & 0.0533 \\
Nile perch fishing net & 0.0094 & 0.0180 \\
\hline
\end{tabular}

Table 3. Distribution of households in SES quintiles during baseline and follow-up surveys

\begin{tabular}{lccccc}
\hline \multirow{2}{*}{ SES quintile } & \multicolumn{2}{c}{ Baseline $(\mathrm{n}=213)$} & & \multicolumn{2}{c}{ Follow-up $(\mathrm{n}=213)$} \\
\cline { 2 - 3 } \cline { 6 - 6 } & Frequency & $(\%)$ & & Frequency & $(\%)$ \\
\hline 1st Most Poor & 53 & 24.9 & & 33 & 15.5 \\
2nd More Poor & 50 & 23.5 & & 37 & 17.4 \\
3rd Poor & 38 & 17.8 & & 45 & 21.1 \\
4th Less poor & 32 & 15.0 & & 53 & 24.9 \\
5th Least poor & 40 & 18.8 & & 45 & 21.1 \\
Total & 213 & 100.0 & & 213 & 100.0 \\
\hline
\end{tabular}




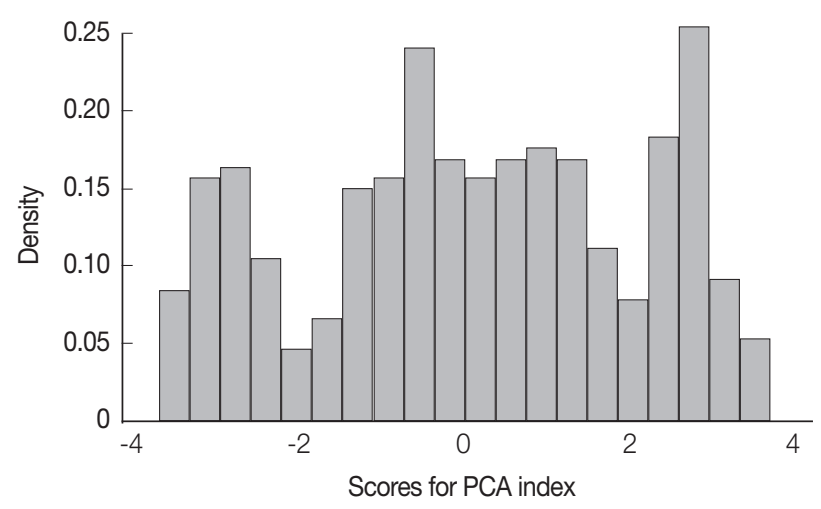

Fig. 1. A histogram for the distribution of PCA scores for baseline and follow-up surveys.

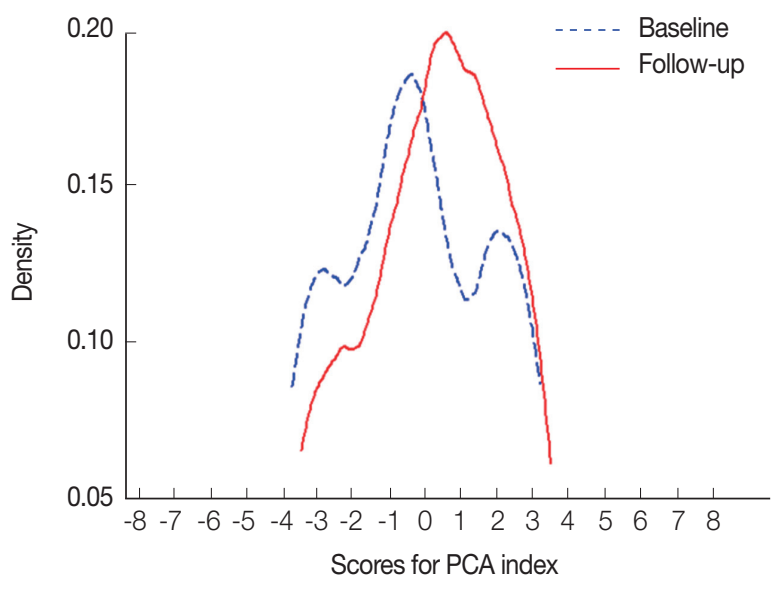

Fig. 2. A density plot of the PCA scores for baseline and followup surveys.

Summary statistics computed from the PCA indices, comparing the results from the baseline and follow-up surveys revealed that the median shifted from -0.437 to 0.500 , the mean shifted from -0.260 to 0.260 and the SD shifted from 2.005 to 1.831 , respectively (Wilcoxon signed rank test prob. $>|z| P<0.0002$ ) and (Student's $t$-test, $P<0.0001$ ).

\section{Significance tests}

PCA: Signrank; Ho: pca_index1 = pca_index2, $\mathrm{z}=-3.7926$, Prob $>|z|=0.0002, t$-test (paired), $P$-value for the test mean (diff) $<0=0.0001$. More importantly, Figs. 1 (a bar chart) and 2 (graph) show the shift in the SES of the study participants between the 2 phases of the study.

\section{DISCUSSION}

This study was set to measure changes in socio-economic status of a community population prior to and 3 years after the introduction of a health intervention by using a household-based asset approach. Findings from this study revealed that it was possible to use household durable assets and housing construction features to measure socio-economic status as it has been demonstrated in previous studies $[5,12,14]$.

However, our study has several limitations worth mentioning. First, we employed a quasi-experimental design studying a single community and comparing the SES of a random sample of households $(n=213)$ before and 3 year after a health intervention. A more rigorous design would entail the inclusion of a control group to overcome potential confounders. However, the use of of household' design in this study is justified, as our investigation was essentially a health education (HE) endeavor aimed at evaluating the effectiveness of a PHAST intervention. A multidimensional focus on the determinants of health and the impossibility of imposing tight environmental controls, or their unacceptability, are inherent features of most health promotion initiatives. The use of a control group, or conducting a randomized controlled trial to evaluate HE initiative would, in this case, have been inappropriate, misleading, and unnecessarily expensive [15,16].

For a better understanding of the impact of health promotion initiatives, evaluators need to use a wide range of qualitative and quantitative methods that extend beyond the narrow parameters of randomized controlled trials. Randomized controlled trials are most effective when the intervention can be delivered and received in a standard way: that is, when variations in delivery and acceptance are minimized. Health promotion programmes can vary greatly in both of these dimensions. Variability may occur, first, in the delivery of an information campaign, implementation of a school/community programme or enforcement of a policy, and, second, in audience attention to or acceptance of campaign messages or participation in a programme. In addition, because health promotion is often a long-term process, frequently involving environmental modifications, attempts to keep environmental conditions constant can undermine the processes that health promotion attempts to influence.

More importantly, a similar 'before/after' design had been used very recently in a study in western Tanzania that investigated the dynamics of SES in the face of schistosomiasis control intervention [12]. In the same vein, other studies in western Côte d'Ivoire investigated the effect of an armed conflict on people's health and changes in their socio-economic posi- 
tion using the same study design $[5,14]$.

Second, the sample size of our study is relatively small (213 households with complete data records from the baseline and the 3-year follow-up cross-sectional surveys) with about 12\% of households lost to follow-up. However, comparing the characteristics of the lost households with those providing complete data showed no significant differences in terms of demographic profile and SES, and hence we believe that our conclusions drawn are valid.

However, it should be noted that the use of an asset approach requires a decision on which assets to collect, or of which assets to select from those available in the dataset being used. The decision to include asset variables in the analysis has a considerable influence on the wealth index than the method used to weigh the data [8]. Care has to be taken not to include either too few or rather too many items in the data set as it may influence the outcome. Moreover, variables such as water and sanitation (for instance, whether a household uses a private tap or a ventilated improved pit latrine) are not solely indicators of household wealth. Rather they are also likely to be direct determinants of the health status of household members $[17,18]$. In our study, we decided to limit the choice of asset indicators to be included in the final analysis to 14 items. We dropped a number of variables related to access to clean water, sanitation, and energy sources, which is in line with studies and experiences made elsewhere $[5,6,8,12,13]$.

Tied to the above methodological issue of choice of asset variables, in a study by Howe and colleagues [19], they concluded that the wealth index is mostly a poor proxy for consumption expenditure, whereas in another study by Hargreaves et al. [20], they urged that more research is needed to increase the validity of measures of SES used in health studies in developing countries.

Findings of this study strongly pointed out towards improvement in SES of the study households after 3 years intervention. Both differences in absolute numbers and percentages in households' asset distribution between pre- and post-intervention surveys were in agreement with PCA weights. The association of positive/negative factor scores with higher/lower SES, respectively, has been found elsewhere $[12,13]$. Hence, the differences in SES of households were real, significant, and accrued over a relatively short time span.

Our data also shows that there has been a shift towards poverty reduction after implementation of the PHAST intervention. In fact, there has been not only decline in poverty but also an increase in wealth in the least poor group. This has implication on equity issues. The intervention seems to have relatively decreased the gap between the most poor and the least poor.

The significance of findings of this study for the scientific community and other stakeholders lies in the fact that with a relatively small sample (213 households) and within a relatively short time (3 years), we were able to demonstrate improvement in SES of a community population following a health education intervention to control schistosomiasis and intestinal worm infections. This implies that while preventive chemotherapy, snail control, sanitation, and clean water supply can all contribute to control of schistosomiasis, the human host can contribute most to the long-term solution of the problem following sustained and appropriate health education and genuine community participation.

The PHAST intervention therefore has succeeded in empowering the study community to become action competent in controlling schistosomiasis, as discussed elsewhere in Tanzania $[11,12]$ and this time also to control intestinal worms. In so doing, it empowered the community also economically. Despite the fact that in the study area there had been other schistosomiasis and intestinal worms control activities, we believe that the improvements observed in the SES within the surveyed households is primarily attributable to the PHAST intervention. Hence, we encourage others to employ the PHAST approach to complement morbidity control through the regular administration of anthelmintic drugs targeting schistosomiasis and other helminthiases.

\section{ACKNOWLEDGMENTS}

We acknowledge Korea International Co-operative Agency (KOICA), for funding the study through the Good Neighbors International, Tanzania Western Chapter. The National Institute for Medical Research (NIMR), Mwanza Centre, and Good Neighbors International, Tanzania Western Chapter are acknowledged for their scientific, technical, and practical support throughout the study period. We thank the people of Kome Island who in one way or another devoted their time to this study.

\section{CONFLICT OF INTEREST}

We have no conflict of interest related to this work. 


\section{REFERENCES}

1. Deaton A, Grosh M. Consumption. In Grosh M, Glewwe P eds, Designing Household Survey Questionnaires for Developing Countries: lessons from ten years of LSMS experience. Washington, USA. The World Bank. 1998, pp. 1-78.

2. Castro-Leal F, Dayton J, Demery L, Mehra K. Public spending on health care in Africa: do the poor benefit? Bull World Health Org 2000; 78: 66-74.

3. Makinen $M$, Waters $H$, Rauch $M$, Almagambetova $N$, Bitran $R$, Gilson L, McIntyre D, Pannarunothai S, Prieto AL, Ubilla G, Ram S. Inequalities in health care use and expenditures: empirical data from eight developing countries and countries in transition. Bull World Health Org 2000; 78: 55-65.

4. Filmer D, Pritchett LH. Estimating wealth effect without expenditure data-or tears: an application to educational enrollments in states of India. Demography 2001; 38: 115-132.

5. Fürst, T, Tschannen AB, Raso G, Acka CA, de Savigny, D, Girardin O, N'Goran EK, Utzinger J. Effect of an armed conflict on relative socioeconomic position of rural households: case study from western Côte d'Ivoire. Emerg Themes Epidemiol 2010; 7: 6.

6. Booysen F, van der Berg S, Burger R, von Maltitz M, du Rand G. Using an asset index to assess trends in poverty in seven sub-Saharan African countries. World Development 2008; 36: 11131130 .

7. Balen J, McManus DP, Li YS, Zhao ZY, Yuan LP, Utzinger J, Williams GM, Li Y, Ren MY, Liu ZC, Zhou J, Raso G. Comparison of two approaches for measuring household wealth via an assetbased index in rural and peri-urban settings of Hunan province, China. Emerg Themes Epidemiol 2010; 7: 7.

8. Howe LD, Hargreaves JR, Huttly SRA. Issues in the construction of wealth indices for measurement of socio-economic position in low-income countries. EmergThemes Epidemiol 2008; 5: 3.

9. National Bureau of Statistics and Ministry of Finance, Dar es Salaam and Office of Chief Government Statistician, Dar es Salaam. President's Office, Finance, Economy and Development Planning, Zanzibar. The 2013 Population and Housing Census.
2013.

10. Wood S, Sawyer R, Simpson-Hebért M. PHAST step-by-step guide: a participatory approach for the control of diarrhoeal diseases. Geneva, Switzerland. World Health Organization (WHO/ EOS/98.3). 1998, pp. 1-137.

11. Mwanga JR, Lwambo NJS. Pre-and post-intervention perceptions and water contact behaviour related to schistosomiasis in north-western Tanzania. Acta Trop 2013; 128: 391-398.

12. Mwanga JR, Lwambo NSJ, Rumisha SF, Vounatsou P, Utzinger J. Dynamics of people's socio-economic status in the face of schistosomiasis control intervention in Ukerewe district, Tanzania. Acta Trop 2013; 128: 399-406.

13. Vyas S, Kumaranayake L. Constructing socio-economic status indices: how to use principal components analysis. Health Policy Plan 2006; 21: 459-468.

14. Fürst T, Raso G, Acka CA, Tschannen AB, N'Goran EK, Utzinger J. Dynamics of socioeconomic risk factors for neglected tropical diseases and malaria in an armed conflict. PLoS Negl Trop Dis 2009; 3: e513.

15. WHO. Health promotion evaluation: recommendation to policy makers. Geneva, Switzerland. World Health Organization. 1998, pp. 1-8.

16. Tones K, Tilford S. Health Promotion: Effectiveness, Efficiency and Equity. 3rd ed. London, UK. Nelson Thornes. 2001.

17. Bartram J, Cairncross S. Hygiene, sanitation, and water: forgotten foundations of health. PLoS Med 2010; 7: e1000367.

18. Ziegelbauer K, Speich B, Mäusezahl D, Bos R, Keiser J, Utzinger J. Effect of sanitation on soil-transmitted helminth infection: systematic review and meta-analysis. PLoS Med 2012; 9 e1001162.

19. Howe LD, Hargreaves JR, Gabrysch S, Huttly SR. Is the wealth index a proxy for consumption expenditure? A systematic review. Int J Equity Health 2009; 8: 29.

20. Hargreaves JR, Morisson LA, Gear JS, Kim JC, Makhubele MB, Porter JD, Watts C, Pronyk PM. Assessing household wealth in health studies in developing countries: a comparison of participatory wealth ranking and survey technique from rural South Africa. Emerg Themes Epidemiol 2007; 4: 4. 
